\title{
National Institute of Mental Health
}

National Cancer Institute

\section{Source}

National Cancer Institute. National Institute of Mental Health. NCI Thesaurus. Code C16894.

An institute within the National Institutes of Health with the mission to transform the understanding and treatment of mental illnesses through basic and clinical research, paving the way for prevention, recovery and cure. 